\title{
Consistent Sobolev Regression via Fuzzy Systems with Overlapping Concepts
}

\author{
Giancarlo Ferrari-Trecate ${ }^{\text {a,1 }}$ Riccardo Rovatti b \\ ${ }^{a}$ INRIA, Domaine de Voluceau \\ Rocquencourt - B.P.105, 78153, Le Chesnay Cedex, France \\ Tel: +33139635921 Fax: +33139635786 \\ e-mail: Giancarlo.Ferrari-Trecate@inria.fr \\ Currently: Dipartimento di Elettronica e Informazione, \\ Politecnico di Milano, Piazza Leonardo da Vinci 32, \\ 20133, Milano, Italy. \\ ${ }^{\mathrm{b}}$ D.E.I.S., Università di Bologna, \\ Viale Risorgimento 2, 40136 Bologna, Italy \\ Tel: +39-051-6443556, Fax +39-051-6443037 \\ E-mail: rrovatti@deis.unibo.it
}

\begin{abstract}
In this paper we propose a new nonparametric regression algorithm based on Fuzzy systems with overlapping concepts. We analyze its consistency properties, showing that it is capable to reconstruct an infinite-dimensional class of function when the size of the noisy dataset grows to infinity. Moreover, convergence to the target function is guaranteed in Sobolev norms so ensuring uniform convergence also for a certain number of derivatives. The connection with Regularization Networks, Bayesian estimation and Tychonov regularization is highlighted.
\end{abstract}

Key words: Learning theory, Fuzzy system models, Regularization Networks, Reproducing kernel Hilbert spaces.

\section{Introduction}

In the last two decades, a large stream of research focused on the universal approximation properties of fuzzy systems. Many investigations (from the classical [5], [17] to [36], [37]) established that any sufficiently regular mapping from inputs to outputs can be approximated,

$\overline{1}$ Corresponding author. 
according to a Lebesgue norm, to any degree of accuracy by fuzzy systems maintaining linguistic interpretability. The case of approximation in the sense of Sobolev norms, that take also the differential behavior into account, was also addressed in [25], [9], [26] and [10].

All these results are based on the availability of noiseless samples of the function to be approximated and show that the error between the fuzzy model and the target can be made less than any prescribed quantity by increasing the number of samples.

A different problem has to be considered when the samples of the target function cannot be thought of as immune to noise. In this case, both problems of approximation and noise filtering have to be addressed at once. What is needed is a regression algorithm which exploits the increasing number of samples to enhance noise rejection as well as to improve approximation accuracy.

Every regression algorithm must incorporate some kind of knowledge about the general features of the target function. Such a knowledge (which in the Bayesian framework is referred to as prior) allows the procedure to discriminate between the information and noise that are present in each sample. Obviously this cannot be done examining each sample alone but is the result of some non-local analysis providing a way to average-out the noise contributions. For instance, in parametric regression the estimators are confined within a finite-dimensional class of functions. In regularization theory $[30,3]$ the prior knowledge is exploited by requiring that the differential features of the estimator possess a prescribed degree of smoothness. It is apparent that the relative weight of priors and data is of major importance. In fact, following the general bias/variance dilemma, any procedure neglecting noise will produce a high-variance model totally dependent on data and with poor generalization capability, while any procedure with limited flexibility (i.e. with too strong prior constraint) will not be able to capture significant features of the model.

In the fuzzy modeling framework, explicit noise filtering mechanisms have been addressed in case of quite strong priors $[28,27,29]$, namely in the case of piecewise-affine target function and models. Here, we propose an implicit scheme relying on milder priors which essentially describe the smoothness of the target function. In order to discuss the properties of this scheme we rely on the well developed theory of the so-called Regularization Networks (RN) and ultimately, on their interpretation both in terms of Tychonov regularization theory [23] and Bayesian estimation [33, 35]. Regression techniques exploiting Tychonov regularization have been the object of intense research in Statistics for the past twenty years. These approaches are also known under the name of "penalized likelihood" or "smoothing spline" methods. The most popular examples are smoothing splines that can be viewed as a particular type of RN [33]. Nowadays, many results are available on the analysis of consistency properties of RN, i.e. on assessing if and how well RNs converge to the target function as the number of noisy data tends to infinity. Typical consistency theorems show that, under suitable assumptions, RN are capable to reconstruct functions in infinite-dimensional Hilbert spaces whose definition is intimately related to the adopted prior. For instance, in [32], consistency has been proved for RNs applied to the reconstruction of functions defined on the interval $[0,1]$ and sampled on a regular grid. In [6], these results have been generalized to 
the case of random sampling but for a fairly limited class of RNs. Consistency properties for the reconstruction of mappings with a multi-dimensional domain have been provided, for instance, in [18], for ANOVA models and in [8] for fairly general classes of RNs.

We show that, analogously to what happens with the activation functions of RNs, a priori knowledge about the smoothness of the unknown function can be easily embedded in a classical Mamdani fuzzy model by choosing the shapes of the membership functions. To this purpose, we introduce Regularization Fuzzy (RF) models and show that many of the positive features of RN extend to RF models.

$\mathrm{RF}$ models are computed through a nonparametric regression technique, where a concept is added for each data point. Quite remarkably, RF estimators can be computed from the noisy data by means of an explicit formula. By construction, RF estimates also borrow from RNs the property that the generalization performance is not spoiled in regions where few samples are collected. In fact, the number of parameters is equal to the number of examples and overfitting is avoided since the weights are not learnt through least squares minimization but by implicitly minimizing a cost functional that penalizes "unlikely" solutions, i.e. non-smooth ones. We point out that, in the noiseless case, regularization techniques have been already proposed in a fuzzy framework to cope with the lack of samples in regions of the domain of the function [16]. Since differential approximation is intrinsically non-local, we choose not to achieve it with models in which concepts (and thus truth functions) are progressively shrunk to cover only a small neighborhood of the point in which they have maximum validity. On the contrary, global approximation is sought by letting the concept overlap and give raise to a more complex (and more accurate) control of the output. To gain a wider generality, we will assume that the domain of the target function is sampled either on a regular grid or according to a uniform probability distribution.

The main result is that RF estimators are consistent in infinite-dimensional Hilbert spaces and the norm used to establish the convergence is a Sobolev norm taking into account as many derivatives as are allowed by the smoothness of the estimator. We highlight that the closed-form expression of RF models is different from the one of RN. As typical of Fuzzy Systems, RF are the ratio between the sum of basis functions and a normalization function. The presence of the denominator does not allow to directly apply consistency results valid for RNs to RF models. In this respect, the consistency theorems that we present hinge on arguments that are substantially different from those used for RNs.

The paper is organized as follows. In Section 2 we recall some basic notions on Reproducing Kernel Hilbert Spaces (RKHSs) that provide the mathematical framework for investigating consistency of RF models. We also introduce the RKHSs associated with Modified Bessel functions of order $r\left(M B_{r}\right)$ which we will use as membership functions. Consistency will be proved in these spaces. Although the more common Gaussian membership functions are not considered in our analysis, we recall that they can be approximated arbitrarily well by $M B_{r}$ functions as $r$ increases. In Section 2.1 the main consistency result for RNs is recalled and, in Section 3, the regression algorithm for RF models is introduced and the consistency of RF estimates stated and discussed. At the end of Section 3 we provide an illustrative 
example. The proof of some technical results and of the main Theorems are reported in the final Appendices.

\section{Preliminaries}

The regression problem addressed in this paper amounts to the estimation of an unknown function $f:[0,1]^{n} \mapsto \mathbb{R}$ from the noisy observations

$$
y_{i}=f\left(\underline{x}_{i}\right)+\epsilon_{i}, i=1, \ldots, N,
$$

where the additive measurements errors $\epsilon_{i}$ are modeled as uncorrelated zero-mean Gaussian random variables with $\operatorname{Var}\left[\epsilon_{i}\right]=\sigma^{2}$. Sampled values are arranged in a column vector $y=$ $\left[y_{1}, \ldots, y_{N}\right]^{\prime}$. The set of the datapoints is $\mathcal{D}_{N}=\left\{\left(\underline{x}_{i}, y_{i}\right), i=1, \ldots, N\right\}$. Throughout the paper we will assume that the points $\underline{x}_{i}$ are extracted in the $n$-dimensional cube $C=[0,1]^{n}$ according to either a deterministic or random sampling schedule.

Assumption 1 The points $\underline{x}_{i}$ lie on a uniform grid in $C$. By letting $K$ be the number of points sampled in each direction on the interval $[0,1]$, one has $N=K^{n}$.

Assumption 2 The points $\underline{x}_{i}$ are generated according the uniform probability distribution over $C$.

Next, we summarize some basic facts about Reproducing Kernel Hilbert Spaces as they will play a key role in deriving the main consistency result. In the sequel, $X \subseteq \mathbb{R}^{n}$ is a Borel set and $G(\underline{x}, \underline{z}): X \times X \mapsto \mathbb{R}$ is a continuous, symmetric, positive-semidefinite function i.e. $\sum_{i=1}^{N} \sum_{j=1}^{N} a_{i} a_{j} G\left(\underline{x}_{i}, \underline{x}_{j}\right) \geq 0, \forall a_{i} \in \mathbb{R}$, for any integer $N$ and for any set of $N$ points $\underline{x}_{i} \in X$. Positive-definite functions are defined by replacing $\geq$ with $>$. We denote the usual Lebesgue spaces of integrable functions defined on $X$ with $\mathbb{L}^{p}(X), p \in[1, \infty)$. It is also assumed that $G(\underline{x}, \underline{z}) \in \mathbb{L}^{2}(X \times X)$. Then, by the Theorems of Mercer, Hilbert, and Schmidt [24], the positive operator $\mathfrak{G}$, defined by

$$
(\mathfrak{G} f)(\underline{x})=\int_{X} G(\underline{x}, \underline{z}) f(\underline{z}) d \underline{z}, \quad f \in \mathbb{L}^{2}(X),
$$

has an $\mathbb{L}^{2}(X)$-complete orthonormal system of eigenfunctions $\left\{\phi_{i}\right\}_{i=1}^{+\infty}$ and corresponding eigenvalues $\left\{\lambda_{i}\right\}_{i=1}^{+\infty}$, with $\lambda_{i}>0$ and $\sum_{i=1}^{+\infty} \lambda_{i}^{2}<+\infty$. Moreover $G(\underline{x}, \underline{z})$ admits the uniformly convergent expansion

$$
G(\underline{x}, \underline{z})=\sum_{i=1}^{+\infty} \lambda_{i} \phi_{i}(\underline{x}) \phi_{i}(\underline{z})
$$

Often, we will refer to $\lambda_{i}$ as "the eigenvalues of $G$ " by omitting the introduction of the corresponding operator $\mathfrak{G}$. Let $\langle\cdot, \cdot\rangle$ be the inner product in $\mathbb{L}^{2}(X)$. The generalized Fourier expansion of $f \in \mathbb{L}^{2}(X)$ is given by

$$
f=\sum_{i=1}^{+\infty} f_{i} \phi_{i}, \quad f_{i} \doteq\left\langle f, \phi_{i}\right\rangle=\int_{X} f(\underline{x}) \phi_{i}(\underline{x}) d \underline{x}, \quad i=1,2, \ldots
$$


We are now in a position to introduce the RKHS associated with $G(\underline{x}, \underline{z})$ [33].

Definition 1. Given a positive-semidefinite function $G(\underline{x}, \underline{z})$, the linear space

$$
\mathcal{H}_{G}(X) \doteq\left\{f: f \in \mathbb{L}^{2}(X), \sum_{i=1}^{+\infty} \frac{f_{i}^{2}}{\lambda_{i}}<+\infty\right\}
$$

equipped with the inner product $\langle f, g\rangle_{G} \doteq \sum_{i=1}^{+\infty} \frac{f_{i} g_{i}}{\lambda_{i}}$ (and associated norm $\|\cdot\|_{G}$ also indicated with $\left.\|\cdot\|_{\mathcal{H}_{G}(X)}\right)$ is a $R K H S$.

The inequality in (4) requires that the generalized Fourier coefficients $f_{i}$ go to zero not too slowly compared to the eigenvalues of $\mathfrak{G}$ and this can be seen as a smoothness condition. The next proposition summarizes some basic properties of RKHSs [2], [23], [33], [22].

Proposition 1. Let $G(\underline{x}, \underline{z})$ be a positive-semidefinite function. Then:

a. $G(\underline{x}, \underline{z}) \in \mathcal{H}_{G}(X), \forall \underline{z} \in X$ and $\langle G(\underline{x}, \underline{z}), f(\underline{z})\rangle_{G}=f(\underline{x}), \forall f \in \mathcal{H}_{G}(X), \forall \underline{x} \in X$ (reproducing property);

b. if $G(\underline{x}, \underline{z})$ has a radial symmetry (i.e. $G(\underline{x}, \underline{z})=g(\|\underline{x}-\underline{z}\|)$ for some $g(\cdot)), \mathcal{H}_{G}(X)$ is dense in $\mathbb{L}^{q}(X)$ for every $q \in[1,+\infty)$ (universal approximation property). Moreover, if $X=\mathbb{R}^{n}$,

$$
\|f\|_{G}^{2}=\int_{\mathbb{R}^{n}} \frac{|\hat{f}(\underline{\omega})|^{2}}{\hat{G}(\underline{\omega})} d \underline{\omega}
$$

where $\hat{f}$ denotes the Fourier transform of $f$.

c. if $f \in \mathcal{H}_{G}(X)$, there exists $\tilde{f} \in \mathcal{H}_{G}\left(\mathbb{R}^{n}\right)$ such that $\|f\|_{\mathcal{H}_{G}(X)}=\left.\|\tilde{f}\|\right|_{\mathcal{H}_{G}\left(\mathbb{R}^{n}\right)}$ and $\left.\tilde{f}\right|_{X}=f$. Moreover, for every $\bar{f} \in \mathcal{H}_{G}\left(\mathbb{R}^{n}\right)$ satisfying $\left.\bar{f}\right|_{X}=f$, it holds $\|\bar{f}\|_{\mathcal{H}_{G}\left(\mathbb{R}^{n}\right)} \geq\|f\| \|_{\mathcal{H}_{G}(X)}$.

The theory of RKHSs is particularly useful when dealing with Regularization Networks [8]. In fact, if $G(\underline{x}, \underline{z})$ is radial, then, roughly speaking, $\mathcal{H}_{G}$ coincides with the set of all functions that can be realized by the associated RN with a proper choice of (possibly infinitely many) centers and weights [33]. The space $\mathcal{H}_{G}$ is usually a non-trivial Hilbert space.

In this work we will use the so called Modified Bessel of order $r\left(M B_{r}\right)$ kernels [31, 34]. In the mono-dimensional case, they are maps $g_{r}: \mathbb{R} \mapsto \mathbb{R}$ defined as

$$
g_{r}(x-z)=\left(\frac{|x-z|}{\tau}\right)^{\nu} \mathcal{K}_{\nu}\left(\frac{|x-z|}{\tau}\right)=\sum_{k=0}^{r-1} a_{k}\left(\frac{|x-z|}{\tau}\right)^{k} \exp \left[-\frac{|x-z|}{\tau}\right]
$$

where $\tau>0, \mathcal{K}_{\nu}$ is the modified Bessel function of order $\nu$ (see e.g. Equation 8.468 in [14]) and $\nu=r-1 / 2$ for integer $r$. The constants $a_{k}$ depend on the order $\nu$ of the Bessel function. For instance, the analytic expression of the Modified Bessel kernels of orders $r=1,2,3$ is: 


$$
\begin{aligned}
& g_{1}(x-z)=\exp \left[-\frac{|x-z|}{\tau}\right], \\
& g_{2}(x-z)=\exp \left[-\frac{|x-z|}{\tau}\right]\left(1+\frac{|x-z|}{\tau}\right) \\
& g_{3}(x-z)=\exp \left[-\frac{|x-z|}{\tau}\right]\left(1+\frac{|x-z|}{\tau}+\frac{1}{3}\left(\frac{|x-z|}{\tau}\right)^{2}\right) .
\end{aligned}
$$

In [20] it is shown that $M B_{r}$ kernels are positive-definite. Although Gaussian kernels (i.e. $\left.g(x-z)=\exp \left[-(x-z)^{2} / 2 \tau\right]\right)$ are not considered in our analysis, it is worth noting that they can be approximated by $M B_{r}$ functions. This can be shown by considering the Fourier Transform of $M B_{r}$ and Gaussian kernels:

$$
\begin{aligned}
\hat{g}_{r}(\omega) & \propto \frac{\tau}{\left(1+\omega^{2} \tau^{2}\right)^{r}} \\
\hat{g}(\omega) & \propto \tau \exp \left[-\frac{\omega^{2} \tau^{2}}{2}\right]
\end{aligned}
$$

Since,

$$
\lim _{r \rightarrow \infty}\left(1+\frac{\omega^{2} \tau^{2}}{2 r}\right)^{-r}=\exp \left[-\frac{\omega^{2} \tau^{2}}{2}\right],
$$

the $M B_{r}$ behaves like the Gaussian kernel, for large $r$, provided that $\tau$ is rescaled accordingly.

As it will be clear in the next Sections, the interest in $M B_{r}$ kernels is motivated by the fact that the eigenvalues $\tilde{\lambda}_{k}$ of $g_{r}:[0,1] \mapsto \mathbb{R}$ asymptotically decrease in a polynomial way. More precisely there exists two positive scalars $l$ and $L, l \leq \mathrm{E}$ such that $l k^{-2 r} \leq \lambda_{k} \leq L k^{-2 r}$. The exact calculation of the $M B_{2}$ eigenvalues is reported in [31].

In the multi-dimensional case, we consider kernels that are tensor products of $M B_{r}$ functions

$$
G_{r}(\underline{x}-\underline{z})=\prod_{i=1}^{n} g_{r}\left(x_{i}-z_{i}\right)
$$

With a little abuse of terminology we shall refer also to $G_{r}(\underline{x}-\underline{z})$ as $M B_{r}$ kernel. It is worth noting that both $G_{r}(\underline{x}-\underline{z})$ and

$$
\bar{G}_{r}(\underline{x}-\underline{z})=\int_{C} G_{r}(\underline{x}-\underline{s}) G_{r}(\underline{s}-\underline{z}) d \underline{s}
$$

are positive-semidefinite functions that define RKHSs $[2,33]$.

Proposition 2. The class of functions

$$
\left\{f: f \in \mathcal{H}_{G_{r}}(C), \sum_{i=1}^{+\infty} \frac{f_{i}^{2}}{\lambda_{i}^{2}}<+\infty\right\}
$$


coincides with $\mathcal{H}_{\bar{G}_{r}}(C)$ and the class

$$
\left\{f(\underline{x})=\prod_{i=1}^{n} f_{i}\left(x_{i}\right), f_{i} \in \mathcal{H}_{g_{r}}([0,1])\right\}
$$

coincides with $\mathcal{H}_{G_{r}}(C)$.

In Appendix A we show that also the eigenvalues of $G_{r}(\underline{x}-\underline{z})$ decrease in a polynomial way. Moreover, if $f, h \in \mathcal{H}_{G_{r}}(C)$ also $f g$ belongs to the same space. We highlight that these properties have been also established in [18,Lemma 2.2] for RKHS that are tensor products of Sobolev spaces equipped with their natural norms.

Next, we clarify the smoothness properties of the functions in $\mathcal{H}_{G_{r}}(C)$ in terms of distributional derivatives [23], [12], [2].

Proposition 3. If $f \in \mathcal{H}_{G_{r}}\left(\mathbb{R}^{n}\right)$, then

$$
\|f\|_{G_{r}}^{2}=\sum_{|\alpha|_{\infty} \leq r} c_{\alpha} \int_{\mathbb{R}^{n}}\left(D^{\alpha} f(\underline{x})\right)^{2} d \underline{x}
$$

where $\alpha=\left(\alpha_{1}, \ldots, \alpha_{n}\right)$ is a multi-index, $|\alpha|_{\infty}=\max _{i=1, \ldots, n} \alpha_{i}, D^{\alpha} f=\frac{\partial^{\sum_{j=1}^{n} \alpha_{j}} f}{\partial x_{1}^{\alpha_{1}} \ldots \partial x_{n}^{\alpha_{n}}}$, and $c_{\alpha}$ are non null scalars that do not depend on $f$. Moreover, if $f \in \mathcal{H}_{G_{r}}(X)$, then

$$
\sum_{|\alpha|_{\infty} \leq r} c_{\alpha} \int_{\mathbb{R}^{n}}\left(D^{\alpha} f(\underline{x})\right)^{2} d \underline{x}<+\infty
$$

Finally, $f \in \mathcal{H}_{G_{r}}(X)\left(f \in \mathcal{H}_{G_{r}}\left(\mathbb{R}^{n}\right)\right)$ implies also that $f$ is $r$ times differentiable and convergence in $\|\cdot\|_{G_{r}}$ implies uniform convergence on $X$ (on $\mathbb{R}^{n}$ ) of the first $r-1$ derivatives.

\subsection{Regularization networks}

One popular method to solve the regression problem is to resort to the so-called Regularization Networks [13] with positive-definite basis function $G(\underline{x}, \underline{z})$. This method considers the following optimization problem

$$
f_{\rho N}(\underline{x}) \doteq \arg \min _{f \in \mathcal{H}_{G}} \sum_{k=1}^{N}\left(y_{k}-f\left(\underline{x}_{k}\right)\right)^{2}+\rho\|f\|_{G}^{2},
$$

where $\rho \geq 0$ is a constant. This is a Tychonov-like regularization problem where the first part of the cost functional weights the sum of the squared residuals and the second one weights the magnitude of $f(\cdot)$ according to the RKHS norm. It is apparent that $\|f\|_{G}$ represents a smoothness constraint. For this reason, the parameter $\rho$ is called regularization parameter. If $\rho=0$, only the sum of the squared residuals is taken in account and $f_{\rho N}(\underline{x})$ fits the $y_{k}$ 
measurements. On the other hand, for $\rho \rightarrow+\infty$, the observations become less important and, in order to minimize $(11), f_{\rho N}(\underline{x})$ converges to zero.

In [13],[15] the explicit formula for $f_{\rho N}(\cdot)$ is provided and $f_{\rho N}(\cdot)$ is termed a regularization network.

Definition 2. Given the data set $\mathcal{D}_{N}$, and a scalar $\rho \geq 0$, the associated regularization network $(R N)$ is the mapping

$$
f_{\rho N}(\underline{x})=\sum_{k=1}^{N} \theta_{i} G\left(\underline{x}, \underline{x}_{i}\right)
$$

where

$$
\begin{aligned}
{\left[\theta_{1} \cdots \theta_{N}\right]^{\prime} } & =\left(G_{N}+\rho I\right)^{-1} \underline{y} \\
{\left[G_{N}\right]_{i j} } & =G\left(\underline{x}_{i}, \underline{x}_{j}\right) .
\end{aligned}
$$

RNs are a class of neural networks and supported by a sound statistical interpretation. According to a Bayesian learning strategy, assume that $f(\cdot)$ in $(1)$ is modeled as a Gaussian $n$ dimensional random field with zero mean and autocovariance function $\mathrm{E}[f(\underline{x}) f(\underline{z})]=G(\underline{x}, \underline{z})$. Then, the Bayes estimate $f_{N}^{B}=\mathrm{E}\left[f \mid \mathcal{D}_{N}\right]$ coincides with $f_{\hat{\rho} N}(\underline{x})$ for $\hat{\rho}=\sigma^{2}$ [33,pag. 19]. RNs are non-parametric estimators since the number of the basis function used is not fixed a priori but it scales with the size $N$ of the data set. If the kernel has radial symmetry, it is immediate to verify that RNs are a special type of Radial Basis Functions neural networks.

In [8] it was proved that RNs are also consistent estimators, in a sense borrowed from statistical estimation theory. In fact, it can be proved that RNs can be expected to converge to the true model (on the set $C$ ) as the size of the data set $\mathcal{D}_{N}$ increases to infinity.

Theorem 1. For $i$ large enough, assume that $l i^{-2 M} \leq \lambda_{i} \leq L i^{-2 m}$ where $l$ and $L$ are two constants satisfying $0<l \leq L<+\infty$ and $m, M$ are two constants in $[1,+\infty)$. Moreover, let $\bar{G}(\underline{x}, \underline{z})$ be defined as $\bar{G}(\underline{x}, \underline{z})=\int_{C} G(\underline{x}, \underline{s}) G(\underline{s}, \underline{z}) d \underline{\text { s. If } \rho \rightarrow+\infty} \rightarrow$ as $N \rightarrow+\infty$ in such a way that $\rho N^{-1 /(2 m+1)} \rightarrow \infty$ and $\rho N^{-1} \rightarrow 0$, then, $\forall f \in \mathcal{H}_{\bar{G}}(C)$, the following properties hold

a. under Assumption 1

$$
\lim _{N \rightarrow+\infty} E_{\epsilon}\left[\left\|f-f_{\rho N}\right\|_{G}^{2}\right]=0 .
$$

b. under Assumption 2, formula (14) holds with probability one.

Theorem 1 deserves some comments. First, note that the error in (14) is measured according to the norm of $\mathcal{H}_{G}(C)$ but it is assumed that $f$ belongs to the smaller space $\mathcal{H}_{\bar{G}}(C)$. The reason for this choice is rather technical and intimately related to the argument for proving Theorem 1 that is very similar to the one adopted in [32,Theorem 2]. Ideally, one would like to assume that $f \in \mathcal{H}_{G}(C)$, but we are not aware of consistency results for RNs proved under this weaker assumption. Second, the fact that we assume $\rho \rightarrow+\infty$ as $N \rightarrow+\infty$ may seem in contradiction with [32, Theorem 2], where the regularization parameter (indicated with $\lambda$ ) is required to converge to zero with $N$. We point out that the optimization problem considered in [32] is similar but not identical to problem (11), the difference being that the sum of 
the squared errors is weighted by $\frac{1}{N}$. Then, one has $\rho=N \lambda$ and Theorem 1 is in complete agreement with [32,Theorem 2]. Finally, the consistency property (14) is more subtle than the usual universal approximation property investigated for various class of neural networks. In fact, it states that RNs filter (asymptotically and on average) the noise $\epsilon_{i}$.

In view of Lemma 1, given in Appendix A, the $M B_{r}$ kernels $G_{r}(\underline{x}-\underline{z})$ satisfy the assumption on the eigenvalues decrease of Theorem 1. More specifically, since from Lemma 1 we have $l^{\prime} k^{-2 r} \leq \lambda_{k} \leq L^{\prime} k^{-2 r\left(\frac{\log _{n} 2}{\log _{n} 2+1}\right)}$, then, according to Theorem 1, the regularization parameter $\rho$ should be increased in a way such that

$$
\rho N^{-\eta} \rightarrow+\infty, \eta=\frac{\log _{n} 2+1}{(2 r+1) \log _{n} 2+1} \quad \text { and } \quad \rho N^{-1} \rightarrow 0
$$

In this case, in view of Proposition 3, the convergence of the RN to the true function $f$ is uniform with all the derivatives up to the $(r-1)$-th order. Then, it is apparent that the choice of smoothness index $r$ should reflect the a priori information that we have about the smoothness of $f$.

\section{Fuzzy Regression and the Consistency Result}

Another strategy for reconstructing $f$ is provided by the use of Fuzzy Systems using $G_{r}(\underline{x}-\underline{z})$ as truth functions for preconditions that result from one-dimensional $M B_{r}$ membership functions put in the "and" relation by means of the real product. In this case, if the most common center of gravity defuzzification is adopted, the estimator is given by

$$
h_{N}(\underline{x})=\frac{\sum_{i=1}^{N} \beta_{i} G_{r}\left(\underline{x}, \underline{x}_{i}\right)}{\sum_{i=1}^{N} G_{r}\left(\underline{x}, \underline{x}_{i}\right)}
$$

In order to prove consistency of such estimators one first has to adopt a suitable strategy for choosing the coefficients $\beta_{i}$.

Definition 3. Given the data set $\mathcal{D}_{N}$, the Fuzzy Regularized (FR) Estimate is given by $h_{\rho N}(\underline{x})$ defined as in (16) where the coefficients $\beta_{i}$ are defined relying on the function

$$
\gamma(\underline{x})=\prod_{j=1}^{n}\left[\Phi\left(x_{j}\right)+\Phi\left(1-x_{j}\right)\right], \quad \Phi(\xi)=\int_{0}^{\xi} g_{r}(t) d t
$$

and the diagonal matrix $H_{N}=\operatorname{diag}\left\{\gamma\left(\underline{x}_{i}\right), i=1, \ldots, N\right\}$ so that

$$
\left[\beta_{1} \cdots \beta_{N}\right]^{\prime}=N G_{N}^{-1} H_{N}\left(I+\rho G_{N}^{-1}\right)^{-1} \underline{y} .
$$

Note that the matrix $G_{N}$ is non-singular since $G_{r}(\underline{x}-\underline{z})$ is a positive-definite function. Formula (18) admits an interesting interpretation. The entries of the vector $\underline{h}=H_{N}(I+$ 
$\left.\rho G_{N}^{-1}\right)^{-1} y$ are the values $f_{\rho N}\left(\underline{x}_{i}\right) \gamma\left(\underline{x}_{i}\right), i=1, \ldots, N$. Therefore, $\underline{c}=G_{N}^{-1} \underline{h}$ gives the coefficients $c_{i}$ of $\tilde{f}(\underline{x})=\sum_{i=1}^{n} c_{i} G_{r}\left(\underline{x}-\underline{x}_{i}\right)$ such that $\tilde{f}$ interpolates the samples $f_{\rho N}\left(\underline{x}_{i}\right) \gamma\left(\underline{x}_{i}\right)$. The less obvious part is the presence of the "correcting factors" $\gamma\left(\underline{x}_{i}\right)$ that, intuitively, balance the asymptotic behavior of the denominator in (16).

The main purpose of this paper is to show that FR estimates are consistent when the distance between the true function and the FR estimate is measured in Sobolev norms. For a given $r \in \mathbb{N} \backslash\{0\}$ we consider the norm

$$
\|f\|_{r}^{2}=\int_{X} \sum_{|\alpha|_{\infty} \leq r}\left(D^{\alpha} f(\underline{x})\right)^{2} d \underline{x},
$$

where $X$ is either $R^{n}$ or $C$ and $\alpha=\left(\alpha_{1}, \ldots, \alpha_{n}\right)$ is a multi-index. Note that if $f \in \mathcal{H}_{G_{r}}(C)$, in view of (10) we have $\|f\|_{r}^{2}<\bar{c}^{-1}\|f\|_{G}, \bar{c}=\min _{|\alpha|_{\infty} \leq r} c_{\alpha}$ and convergence with respect to $\|\cdot\|_{r}$ is implied by convergence with respect to $\|\cdot\|_{G_{r}}$. We are now in a position to state the main result of the paper.

Theorem 2. Let the assumptions of Theorem 1 hold. Then, $\forall f \in \mathcal{H}_{\bar{G}_{r}}(C)$, the following properties hold

a. under Assumption 1

$$
\lim _{N \rightarrow+\infty} \mathrm{E}_{\underline{\epsilon}}\left[\left\|f-h_{\rho N}\right\|_{r-1}^{2}\right]=0 .
$$

b. under Assumption 2, formula (20) holds with probability one.

The proof of Theorem 2 is reported in the Appendix. Analogously to Theorem 1 it states that the FR estimate converges to the true function uniformly with the derivatives up to $(r-1)$-th order. For the choice of $\rho$, the same asymptotic conditions given in (15) hold.

Note that, in the statement of Theorem 2, the parameter $\tau$ (specifying the width of the $M B_{r}$ function, see formula (5)) is fixed and does not scale with $N$.

However, when using FR estimators on a finite dataset, $\tau$ (and/or the regularization parameter $\rho$ are usually unknown and they must be estimated from the collected data points. If a validation set is available, cross validation techniques could be used for their tuning. Alternatively, for fixed $\tau$, one can exploit criteria like Maximum Likelihood [19] or Generalized Cross Validation [7] that are widely used for tuning the smoothness parameter of RNs. Another possibility is or resort to Monte Carlo methods for the simultaneous tuning of $\tau$ and $\rho[21]$. Of course, such procedures are not optimal for FR estimators, since they are tailored to RNs and, for a finite amount of data, one has that $h_{\rho N} \neq f_{\rho N}$. However, in many practical experiments, we found that the algorithm for tuning the parameters of RNs give very similar results when applied to FR models.

In the remainder of this Section we provide an example to illustrate the properties or FR estimators. We choose $n=2$ and consider the target function $f\left(x_{1}, x_{2}\right)=\sin \left(2 \pi x_{1}\right) \cos \left(2 \pi x_{2}\right) \in$ $\mathbb{C}^{\infty}$. The samples $\underline{x}_{i}$ have been extracted according to Assumption 2. The variance of the noise corrupting the output samples is $\sigma^{2}=0.01$. We consider fuzzy systems based on $M B_{2}$ 


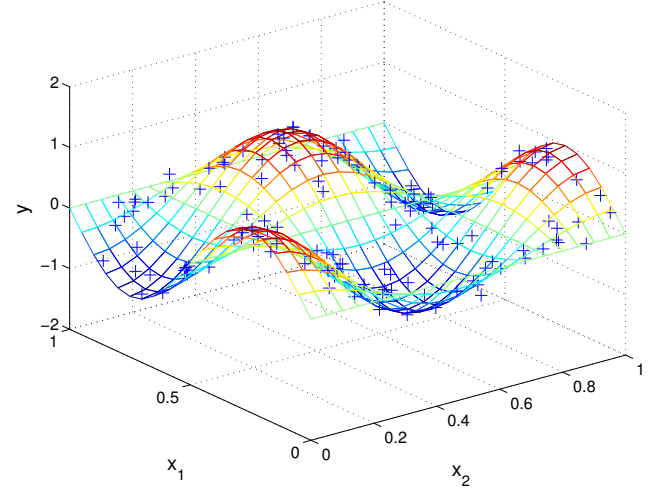

a

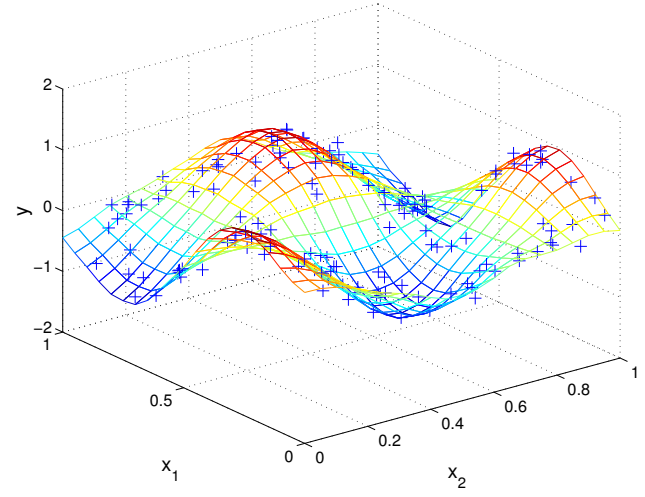

$\mathrm{b}$

Fig. 1. Regression with FR models: a) Target function and collected datapoints; b) FR estimate;

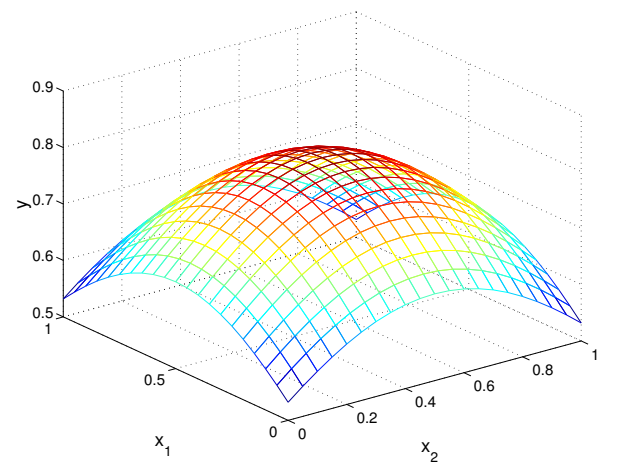

Fig. 2. The correcting function $\gamma(\underline{\mathrm{x}})$.

membership functions with $\tau=0.5$. As mentioned in Section 2.1, we exploit the Bayesian interpretation of the regularization parameter $\rho$ and set it equal to the noise variance.

In the first experiment we collected $N=144$ data points. In Figure 1-a the target function and the data points are shown. Trivial visual inspection shows that the regression results are good both in terms of punctual and differential behavior. The correcting function $\gamma(\underline{x})$, defined by (17), is depicted in Figure 2 .

In a second experiment, in order to study the convergence property of FR estimators, we computed the quantities

$$
\begin{aligned}
& E_{N}^{0}=\mathrm{E}\left[\int_{[0,1]^{2}}\left(f-h_{\rho N}\right)^{2},\right] \\
& E_{N}^{1}=\mathrm{E}\left[\int_{[0,1]^{2}}\left[\frac{\partial}{\partial x_{1}}\left(f-h_{\rho N}\right)\right]^{2}+\left[\frac{\partial}{\partial x_{2}}\left(f-h_{\rho N}\right)\right]^{2}\right]
\end{aligned}
$$

for data sets of sizes $N=7^{2}, 8^{2}, 9^{2}, 10^{2}, 11^{2}$. The expectations have been approximated by averaging the integrals in (21) and (22) over five different experiments for each number of 


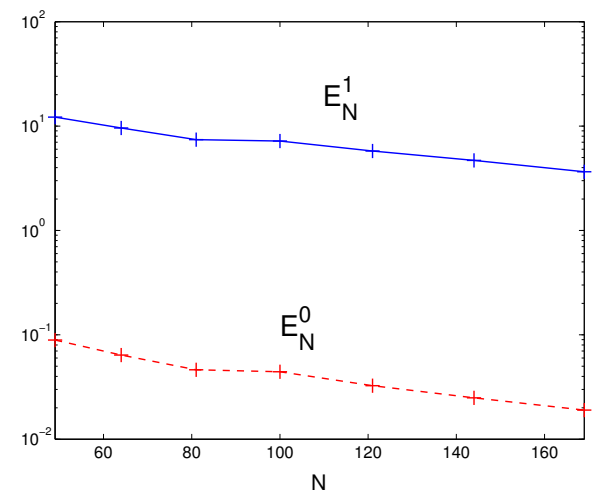

Fig. 3. Convergence of 0 th and 1st order components of $\left\|f-h_{\rho N}\right\|_{1}$.

data points.

The results are drawn in Figure 3. Since $\left\|f-h_{\rho N}\right\|_{1}=E_{N}^{0}+E_{N}^{1}$, despite the different relative magnitude of the terms, convergence is evident in all the components of the error norm.

\section{Conclusions}

In this work we proposed a regression algorithm for fuzzy systems that enjoys consistency property in Sobolev norms. Non-locality of the concepts plays a key role in consistent identification in presence of noise, as it has been already proved for RNs [8]. An interesting problem is to design data-based algorithm for the automatic tuning of the smoothness parameter $\rho$. In fact, in the RN framework, statistical tests like Generalized Cross Validation or Maximum Likelihood are commonly adopted for the choice of the regularization parameter. The derivation of similar algorithms for FR estimators will be topic of future investigations.

\section{Acknowledgments}

The authors thank Dr. I. Longhi for the help in proving Lemma 1. This research has been supported by the the MURST Project "Identification and Control of Industrial Systems".

\section{Appendix A}

In this Appendix we prove two key properties of the $M B_{r}$ kernels $G_{r}$ defined in (7). The next Lemma shows that if $G$ is a tensor product of mono-dimensional kernels with polynomially decreasing eigenvalues, then $G$ inherits the same property. As a consequence, one deduces that the eigenvalues of $G_{r}$ decay in a polynomial way. 
Lemma 1. Let $l, L$ and $c$ be three constant such that $0<l \leq L<+\infty, c>0$ and $l k^{-c} \leq$ $\tilde{\lambda}_{k} \leq L k^{-c}$, where $\tilde{\lambda}_{k}$ are the eigenvalues of a positive-semidefinite function $g(x, z): \mathbb{R}^{2} \mapsto \mathbb{R}$. Then, there exists $0<l^{\prime} \leq L^{\prime}<+\infty$ such that the eigenvalues $\lambda_{k}$ of $G(\underline{x}, \underline{z})=\prod_{i=1}^{n} g\left(x_{i}, z_{i}\right)$, $n>1$, for $k$ large enough satisfy

$$
l^{\prime} k^{-c} \leq \lambda_{k} \leq L^{\prime} k^{-c\left(\frac{\log _{n} 2}{\log _{n} 2+1}\right)} .
$$

Proof. In view of $(2), g(x, z)=\sum_{i=1}^{+\infty} \tilde{\lambda}_{i} \phi_{i}(x) \phi_{i}(z)$ and

$$
G(\underline{x}, \underline{z})=\prod_{i=1}^{n} \sum_{j_{i}=1}^{\infty} \tilde{\lambda}_{j_{i}} \phi_{j_{i}}\left(x_{i}\right) \phi_{j_{i}}\left(y_{i}\right)=\sum_{j_{1}=1}^{\infty} \cdots \sum_{j_{n}=1}^{\infty} \prod_{i=1}^{n} \tilde{\lambda}_{j_{i}} \prod_{i=1}^{n} \phi_{j_{i}}\left(x_{i}\right) \prod_{i=1}^{n} \phi_{j_{i}}\left(y_{i}\right) .
$$

Then, the eigensystem of $G$ is given by the eigenvalues $\prod_{i=1}^{n} \tilde{\lambda}_{j_{i}}$ and by the eigenfunctions $\prod_{i=1}^{n} \phi_{j_{i}}$ for every set of indexes $j_{1}, \ldots, j_{n}$. We consider the multi-index $J=\left(j_{1}, \ldots, j_{n}\right)$ and indicate with $\lambda_{J}$ the product $\prod_{i=1}^{n} \tilde{\lambda}_{j_{i}}$.

To prove the Lemma we first need to sort the eigenvalues $\lambda_{J}$ in a proper way. For an integer $p$ we define the set of multi-indexes $E_{p}=\left\{J: \prod_{i=1}^{n} j_{i}=p\right\}$. We sort the $\lambda_{J}$ according the following rule: For two indexes $i^{\prime}, i^{\prime \prime}$, if $\lambda_{i^{\prime}}=\lambda_{J^{\prime}}$ and $\lambda_{i^{\prime \prime}}=\lambda_{J^{\prime \prime}}$ where $J^{\prime} \in E_{p^{\prime}}, J^{\prime \prime} \in E_{p^{\prime \prime}}$ and $p^{\prime}<p^{\prime \prime}$, then $i^{\prime}<i^{\prime \prime}$. Moreover, the eigenvalues $\lambda_{J}$ such that the multi-index $J$ belongs to the same $E_{p}$ are sorted in non increasing order. For every $J \in E_{p}$, by using the bounds on $\tilde{\lambda}_{k}$, we have

$$
l^{n} p^{-c} \leq \lambda_{J} \leq L^{n} p^{-c}
$$

and the number of $\lambda_{J}$ for which the bounds (25) holds is equal to the cardinality of $E_{p}$. Since the number of prime numbers (counted with their multiplicity) that factor an integer $p$ is less than $\log _{2} p$ and bigger than one, we have that

$$
n \leq \operatorname{card}\left(E_{p}\right) \leq n^{\log _{2} p}=p^{\frac{1}{\log _{n} 2}}
$$

The next aim is to look for an upper bound $U(k) \geq \lambda_{k}$. Note that every monotone decreasing $U(k)$ satisfying

$$
U\left(\sum_{i=1}^{p}\left\lfloor i^{\frac{1}{\log _{n} 2}}\right\rfloor\right) \geq L^{n} p^{-c}, \quad \forall p \in \mathbb{N}
$$

is an upper bound in view of the ordering on the $\lambda_{k}$ previously introduced and of the bound (25). To see this, note that $\sum_{i=1}^{p}\left\lfloor i^{\frac{1}{\log _{n} 2}}\right\rfloor \geq \sum_{i=1}^{p} \operatorname{card}\left(E_{i}\right)$. On the other hand $\sum_{i=1}^{p} i^{\frac{1}{\log _{n} 2}} \leq$ $a(p+1)^{\frac{1}{\log _{n} 2}+1}$ where $a \in \mathbb{R}^{+}$is a suitably defined constant that does not depend on $p$. It follows that if $U\left(a(p+1)^{\frac{1}{\log _{n} 2}+1}\right) \geq L^{n} p^{-c}$, then $U(k)$ is also an upper bound to $\lambda_{k}$. It is easy to verify that the function

$$
U(k)=L^{n}\left[\left(\frac{k}{a}\right)^{\left(\frac{\log _{n} 2}{\log _{n} 2+1}\right)}-1\right]^{-c}
$$


satisfies the above requirement. To what concerns the lower bound, we look for a monotone function $L(k)$ such that $L(n p) \leq l^{n} p_{c}$. Then $L(k)=l^{n}\left(\frac{k}{n}\right)^{-c}$ is a possible choice. By summarizing the previous results, we have

$$
l^{n}\left(\frac{k}{n}\right)^{-c} \leq \lambda_{k} \leq L^{n}\left[\left(\frac{k}{a}\right)^{\left(\frac{\log _{n} 2}{\log _{n} 2+1}\right)}-1\right]^{-c}
$$

and, for $k$ large,

$$
l^{n}\left(\frac{k}{n}\right)^{-c} \leq \lambda_{k} \leq L^{n}\left(\frac{k}{a}\right)^{-c\left(\frac{\log _{n} 2}{\log _{n} 2+1}\right)} .
$$

To obtain formula (23) it is enough to choose $L^{\prime}=L^{n} / a^{-c\left(\frac{\log _{n} 2}{\log _{n} 2+1}\right)}$ and $l^{\prime}=l^{n} / n^{c}$.

Next, we show that the spaces $\mathcal{H}_{G_{r}}(C)$ is closed under function multiplication.

Proposition 4. If $f \in \mathcal{H}_{G_{r}}(C)$ and $h \in \mathcal{H}_{G_{r}}(C)$, then fh $\in \mathcal{H}_{G_{r}}(C)$.

Proof. Consider the monodimensional case, i.e. $C=[0,1]$. From point (c) of Proposition 1 , there exists $\tilde{f} \in \mathcal{H}_{g_{r}}(\mathbb{R})$ such that $\|f\|_{\mathcal{H}_{g_{r}}(C)}=\|\tilde{f}\|_{\mathcal{H}_{g_{r}}(\mathbb{R})}$ and $\left.\tilde{f}\right|_{C}=f$. We denote with $\tilde{h}$ the analogous extension for $h$. Let us define the Sobolev space [1]

$$
H^{r}=\left\{f: \mathbb{R} \mapsto \mathbb{R} \mid D^{\nu} f \text { is absolutely continuous, } \nu=0,1, \ldots, r-1, D^{r} f \in \mathbb{L}^{2}\right\}
$$

equipped with the norm

$$
\|\tilde{f}\|_{H^{r}}^{2}=\int_{\mathbb{R}}|\hat{f}(\omega)|^{2}\left(1+\omega^{2}\right)^{r} d \omega
$$

where $\hat{f}$ is the Fourier transform of $\tilde{f}$. We show that $\tilde{f} \in H^{r} \Leftrightarrow \tilde{f} \in \mathcal{H}_{g_{r}}(\mathbb{R})$. From point (b) of Proposition 1 and (6) one has

$$
\tilde{f} \in \mathcal{H}_{g_{r}}(\mathbb{R}) \Rightarrow \tau^{-1} \int_{\mathbb{R}}|\hat{f}(\omega)|^{2}\left(1+\tau^{2} \omega^{2}\right)^{r} d \omega \leq+\infty
$$

By using the change of variables $y=\tau \omega$, the last integral implies that $\|\tilde{f}(\tau t)\|_{H^{r}}^{2} \leq+\infty$ and hence $f(t) \in H^{r}$. A similar argument shows also that $\tilde{f} \in H^{r} \Rightarrow \tilde{f} \in \mathcal{H}_{g_{r}}(\mathbb{R})$. Since it is known that $\tilde{f}, \tilde{h} \in H^{r} \Rightarrow \tilde{f} \tilde{h} \in H^{r}$ (see [1]) one also has that $\tilde{f} \tilde{h} \in \mathcal{H}_{g_{r}}(\mathbb{R})$.

By taking the restrictions to $C$, we obtain $f h \in \mathcal{H}_{g_{r}}(C)$. Finally, from Proposition 2, we have that also in the multi-dimensional case (i.e. $\left.C=[0,1]^{n}\right)$, it holds $f h \in \mathcal{H}_{G_{r}}(C)$.

\section{Appendix B}

We start introducing a Lemma that clarifies the asymptotic behavior of the approximator 


$$
\begin{aligned}
R_{N}(\underline{x}, \underline{\epsilon}) & =\sum_{k=1}^{N} b_{k, N}(\underline{\epsilon}) G\left(\underline{x}-\underline{x}_{k}\right) \\
\underline{b}_{N}(\underline{\epsilon}) & =G_{N}^{-1}\left[f_{N}\left(\underline{x}_{1}, \underline{\epsilon}\right), \ldots, f_{N}\left(\underline{x}_{N}, \underline{\epsilon}\right)\right]^{\prime}
\end{aligned}
$$

where $\underline{\epsilon} \in \mathbb{R}^{N}$ is a random vector, $G$ is a positive-definite function and $G_{N}$ is defined as in (13). Note that, as $N$ increases, the functions $f_{N}$ to be approximated, change.

Lemma 2. Let $f(\underline{x}) \in \mathcal{H}_{G}(C)$ and $f_{N}(\underline{x}, \underline{\epsilon})$ be a sequence of functions dependent on the random vectors $\underline{\epsilon} \in \mathbb{R}^{N}$ such that

1. $f_{N}(\underline{x}, \underline{\epsilon}) \in \mathcal{H}_{G}(C), \forall \underline{\epsilon} \in \mathbb{R}^{N}$,

2. $\lim _{N \rightarrow+\infty} \mathrm{E}_{\underline{\epsilon}}\left[\left\|f_{N}(\underline{x}, \underline{\epsilon})-f(\underline{x})\right\|_{G}^{2}\right]=0$.

Define $R_{N}(\underline{x}, \underline{\epsilon})$ as in (29). Then,

a. under Assumption 1,

$$
\lim _{N \rightarrow+\infty} \mathrm{E}_{\underline{\epsilon}}\left[\left\|R_{N}(\underline{x}, \underline{\epsilon})-f(\underline{x})\right\|_{G}^{2}\right]=0
$$

b. under Assumption 2, formula (33) holds with probability one.

Proof. By using triangle inequality,

$$
\mathrm{E}_{\underline{\epsilon}}\left[\left\|R_{N}(\underline{x}, \underline{\epsilon})-f(\underline{x})\right\|_{G}^{2}\right] \leq \mathrm{E}_{\underline{\epsilon}}\left[\left\|f_{N}(\underline{x}, \underline{\epsilon})-f(\underline{x})\right\|_{G}^{2}\right]+\mathrm{E}_{\underline{\epsilon}}\left[\left\|R_{N}(\underline{x}, \underline{\epsilon})-f_{N}(\underline{x})\right\|_{G}^{2}\right],
$$

and since $\mathrm{E}_{\epsilon}\left[\left\|f_{N}(\underline{x}, \underline{\epsilon})-f(\underline{x})\right\|_{G}^{2}\right]$ converges to zero, we concentrate on the second term. In the sequel $\bar{V} D V^{\prime}=G_{N}, D=\operatorname{diag}\left\{\lambda_{\mathrm{iN}}\right\}, i=1, \ldots N$, is the singular value decomposition of $G_{N}$. Moreover, $V=\left[\begin{array}{llll}v_{1 N} & v_{2 N} & \ldots & v_{N N}\end{array}\right]$, where $v_{i N}=\left[\begin{array}{lll}v_{i N}(1) & v_{i N}(2) \ldots v_{i N}(N)\end{array}\right]^{\prime}$ denotes the $i$-th eigenvector of $G_{N}$.

Point a. Let Assumption 1 holds. Then, as in [32] we will exploit the fact that, for large $N$, one may set

$$
\begin{aligned}
\sum_{k=1}^{N} G\left(\underline{x}_{i}, \underline{x}_{k}\right) \phi_{j}\left(\underline{x}_{k}\right) & \simeq N \lambda_{j} \phi_{j}\left(\underline{x}_{i}\right), \quad N \lambda_{i} \simeq \lambda_{i N}, \\
\frac{1}{\sqrt{N}} \phi_{i}\left(\underline{x}_{j}\right) & \simeq v_{i N}(j), \quad N \tilde{f}_{i N}^{2} \simeq f_{i N}^{2}, \quad \tilde{f}_{i N} \doteq \sum_{j=1}^{N} v_{i N}(j) f_{N}\left(\underline{x}_{j}\right)
\end{aligned}
$$

where $f_{i N}$ are the Fourier coefficients (defined as in (3)) of $f_{N}$. By direct calculation one obtains

$$
\left\|f-r_{N}\right\|_{G}^{2}=\left\|\sum_{k=1}^{+\infty} f_{k N} \phi_{k}(\underline{x})-\sum_{k=1}^{N}\left(\sum_{j=1}^{N} \frac{G\left(\underline{x}, \underline{x}_{j}\right) v_{k N}(j)}{\lambda_{k N}}\right)\left(\sum_{i=1}^{N} v_{k N}(i) f_{N}\left(\underline{x}_{i}\right)\right)\right\|_{G}^{2},
$$

and using the asymptotic approximations, for $N$ large it holds 


$$
\begin{aligned}
\sum_{j=1}^{N} \frac{G\left(\underline{x}, \underline{x}_{j}\right) v_{k N}(j)}{\lambda_{k N}} & \simeq \frac{1}{\sqrt{N}} \phi_{k}(\underline{x}), \sum_{i=1}^{N} v_{k N}(i) f_{N}\left(\underline{x}_{i}\right) \simeq \sqrt{N} f_{k N}, \\
\left\|f-r_{N}\right\|_{G}^{2} & \simeq\left\|\sum_{k=N+1}^{+\infty} f_{k N} \phi_{k}(\underline{x})\right\|_{G}^{2}=\sum_{k=N+1}^{+\infty} \frac{f_{k N}^{2}}{\lambda_{k}} .
\end{aligned}
$$

By using the inequality $(a+b)^{2} \leq 2 a^{2}+2 b^{2}$

$$
\mathrm{E}\left[\sum_{k=N+1}^{+\infty} \frac{f_{k N}^{2}}{\lambda_{k}}\right] \leq 2 \mathrm{E}\left[\sum_{k=N+1}^{+\infty} \frac{\left(f_{k N}-f_{k}\right)^{2}}{\lambda_{k}}\right]+2 \mathrm{E}\left[\sum_{k=N+1}^{+\infty} \frac{f_{k}^{2}}{\lambda_{k}}\right]
$$

where $f_{k}$ are the Fourier coefficients of $f$. The second term in the r.h.s of (36) goes to zero, since $f \in \mathcal{H}_{G}$. Moreover, from the definition of $\|\cdot\|_{G}$ it follows

$$
\mathrm{E}\left[\sum_{k=N+1}^{+\infty} \frac{\left(f_{k N}-f_{k}\right)^{2}}{\lambda_{k}}\right] \leq \mathrm{E}\left[\left\|f_{N}-f\right\|_{G}^{2}\right]
$$

that goes to zero by assumption.

Point $b$. Under Assumption 2, by Birkov Ergodic Theorem [4] , the approximations (34)-(35) hold for almost every realization of the sampling process. Then, the proof proceeds in the same way as in point a.

Lemma 3. The function $\gamma(\underline{x})$ defined by (17) belongs to the space $\mathcal{H}_{G_{r}}\left(\mathbb{R}^{n}\right)$. Moreover its Fourier Transform $\hat{\gamma}(\underline{\omega})$ belongs to $\mathbb{L}^{1}\left(\mathbb{R}^{n}\right)$.

Proof. Consider the scalar functions

$$
\gamma^{\prime}(x)=\tau\left[\Phi\left(\frac{x}{\tau}\right)+\Phi\left(\frac{x-1}{\tau}\right)\right], \quad \Phi(\xi)=\int_{0}^{\xi} g_{r}(t) d t
$$

where $g_{r}$ is defined in (5) and which are such that $\tau^{n} \gamma(\underline{x} \backslash \tau)=\prod_{j=1}^{n} \gamma^{\prime}\left(x_{j}\right)$ and $G(\underline{x}, \underline{z})=$ $\prod_{j=1}^{n} G^{\prime}\left(x_{j}, z_{j}\right)$. Note that $D^{1} \gamma^{\prime}(x)=g_{r}^{\prime}(x)-g_{r}^{\prime}(x-1)$ so that, by taking the Fourier Transform, and by using (6), one has

$$
\hat{\gamma^{\prime}}(\omega) \propto \frac{\tau}{\left(1+\omega^{2} \tau^{2}\right)^{r}} \frac{1-e^{-\mathbf{i} \omega}}{\mathbf{i} \omega} .
$$

With this and (9) we have

$$
\left\|\gamma^{\prime}\right\|_{g_{r}} \propto \int_{\mathbb{R}} \frac{\tau}{\left(1+\omega^{2} \tau^{2}\right)^{r}} \frac{2-2 \cos (\omega)}{\omega^{2}} d \omega
$$


which eventually converges showing that $\gamma^{\prime} \in \mathcal{H}_{g_{r}}$. Yet, we know from Proposition 2 that the whole $\gamma$ (which is the tensor product of the $\gamma^{\prime}$ functions) belongs to a RKHS whose kernel is the tensor product of $g_{r}$, i.e. $\mathcal{H}_{G_{r}}$.

To what concerns the second part of the thesis, note that

$$
\|\hat{\gamma}\|_{\mathbb{L}^{1}}=\int_{\mathbb{R}^{n}} \prod_{i=1}^{n} \frac{\tau}{\left(1+\omega_{i}^{2} \tau^{2}\right)^{r}} \frac{\left(2-2 \cos \left(\omega_{i}\right)\right)^{\frac{1}{2}}}{\omega} d \underline{\omega}
$$

and then, by direct calculation, $\|\hat{\gamma}\|_{\mathbb{L}^{1}}<+\infty$

Corollary 1. Let $G=G_{r}, f$ and $f_{N}$ be defined as in Lemma 2. Assume also that (31) and (32) hold and consider the approximator

$$
\begin{aligned}
\bar{R}_{N}(\underline{x}, \underline{\epsilon}) & =\sum_{k=1}^{N} \bar{b}_{k, N}(\underline{\epsilon}) G_{r}\left(\underline{x}-\underline{x}_{k}\right) \\
\underline{b}_{N}(\underline{\epsilon}) & =G_{N}^{-1} H_{N}\left[f_{N}\left(\underline{x}_{1}, \underline{\epsilon}\right), \ldots, f_{N}\left(\underline{x}_{N}, \underline{\epsilon}\right)\right]^{\prime} .
\end{aligned}
$$

Then,

a. under Assumption 1

$$
\lim _{N \rightarrow+\infty} \mathrm{E}_{\underline{\epsilon}}\left[\left\|\bar{R}_{N}(\underline{x}, \underline{\epsilon})-f(\underline{x}) \gamma(\underline{x})\right\|_{G_{r}}^{2}\right]=0
$$

b. under Assumption 2 formula (40) holds with probability one with respect the joint distribution of $\left(\underline{x}_{1}, \underline{x}_{2}, \ldots, \underline{x}_{N}, \ldots\right)$.

Proof. Note that

$$
H_{N}\left[f_{N}\left(\underline{x}_{1}, \underline{\epsilon}\right), \ldots, f_{N}\left(\underline{x}_{N}, \underline{\epsilon}\right)\right]^{\prime}=\left[\gamma\left(\underline{x}_{1}\right) f_{N}\left(\underline{x}_{1}, \underline{\epsilon}\right), \ldots, \gamma\left(\underline{x}_{N}\right) f_{N}\left(\underline{x}_{N}, \underline{\epsilon}\right)\right]^{\prime}
$$

so that the goal of the proof is to apply Lemma 2 to the sequence $\bar{f}_{N}(\underline{x}, \underline{\epsilon})=\gamma(\underline{x}) f_{N}(\underline{x}, \underline{\epsilon})$ with $G=G_{r}$. Note that, from Lemma $3, \gamma \in \mathcal{H}_{G}(C)$ and from Proposition 4, the products $\gamma(\underline{x}) f_{N}(\underline{x}, \underline{\epsilon})$ and $\gamma(\underline{x}) f(\underline{x})$ belong to $\mathcal{H}_{G}(C)$.

The sequence $\bar{f}_{N}(\underline{x}, \underline{\epsilon})$ satisfies assumption (31) of Lemma 2. To what concerns assumption (32), for the sequence $\bar{f}_{N}(\underline{x}, \underline{\epsilon})$, we have to prove that $\mathrm{E}\left[\left\|\gamma f_{N}-\gamma f\right\|_{G_{r}}\right]$ converges to zero.

Let $p_{N}(\underline{x}, \underline{\epsilon}) \doteq f_{N}(\underline{x}, \underline{\epsilon})-f(\underline{x})$. The functions $\underline{x} \mapsto p_{N}(\underline{x}, \underline{\epsilon})$ are in $\mathcal{H}_{G_{r}}(C)$ for every $\underline{\epsilon}$, and from point (c) of Proposition 1, there exists $\tilde{p}(\underline{x}, \underline{\epsilon}) \in \mathcal{H}_{G_{r}}\left(\mathbb{R}^{n}\right)$ such that $\left.\tilde{p}\right|_{C}=p$ and

$$
\mathrm{E}_{\underline{\epsilon}}\left[\left\|\tilde{p}_{N}\right\|_{\mathcal{H}_{G_{r}}\left(\mathbb{R}^{n}\right)}\right]=\mathrm{E}_{\underline{\epsilon}}\left[\left\|p_{N}\right\|_{\mathcal{H}_{G_{r}}(C)}\right]
$$

We can use the Fourier transforms as in point (b) of Proposition 1 to derive 


$$
\begin{aligned}
\mathrm{E}_{\underline{\epsilon}}\left[\left\|\gamma \tilde{p}_{N}\right\|_{\left.\mathcal{H}_{G_{r}}\left(\mathbb{R}^{n}\right)\right]}\right. & =\mathrm{E}_{\underline{\epsilon}}\left[\int_{\mathbb{R}^{n}} \frac{\left|\left(\hat{\gamma} \otimes \hat{p}_{N}\right)(\underline{\omega})\right|^{2}}{\hat{G}_{r}(\underline{\omega})} d \underline{\omega}\right]= \\
& =\mathrm{E}_{\underline{\epsilon}}\left[\left\|\left(\hat{G}_{r}\right)^{-1}\left|\hat{\gamma} \otimes \hat{p}_{N}\right|^{2}\right\|_{\mathbb{L}^{1}}\right]
\end{aligned}
$$

where $\otimes$ denotes the convolution operator. By using arguments similar to the ones employed in the proof of Young's Theorem [1], one can derive the inequalities

$$
\begin{aligned}
& \left\|\left(\hat{G}_{r}\right)^{-1}\left|\hat{\gamma} \otimes \hat{p}_{N}\right|^{2}\right\|_{\mathbb{L}^{1}} \leq\|\hat{\gamma}\|_{\mathbb{L}^{1}}\left\|\left(\hat{G}_{r}\right)^{-1}\left|\hat{p}_{N}\right|^{2} \otimes|\hat{\gamma}|\right\|_{\mathbb{L}^{1}} \\
& \left\|\left(\hat{G}_{r}\right)^{-1}\left|\hat{p}_{N}\right|^{2} \otimes|\hat{\gamma}|\right\|_{\mathbb{L}^{1}} \leq\|\hat{\gamma}\|_{\mathbb{L}^{1}}\left\|\left(\hat{G}_{r}\right)^{-1}\left|\hat{p}_{N}\right|^{2}\right\|_{\mathbb{L}^{1}} .
\end{aligned}
$$

Note that $\hat{\gamma} \in \mathbb{L}^{1}$ as stated in Lemma 3. By substituting (43) in (42), we obtain

$$
\begin{aligned}
\mathrm{E}_{\underline{\epsilon}}\left[\left\|\gamma \tilde{p}_{N}\right\|_{\left.\mathcal{H}_{G_{r}}\left(\mathbb{R}^{n}\right)\right]}\right. & \leq \mathrm{E}_{\underline{\epsilon}}\left[\|\hat{\gamma}\|_{\mathbb{L}^{1}}^{2}\left\|\left(\hat{G}_{r}\right)^{-1}\left|\hat{p}_{N}\right|^{2}\right\|_{\mathbb{L}^{1}}\right] \\
& =\|\hat{\gamma}\|_{\mathbb{L}^{1}}^{2} \mathrm{E}_{\underline{\epsilon}}\left[\left\|\left(\hat{G}_{r}\right)^{-1}\left|\hat{p}_{N}\right|^{2}\right\|_{\mathbb{L}^{1}}\right] .
\end{aligned}
$$

From (32) and (41), we have that $\mathrm{E}_{\underline{\epsilon}}\left[\left\|\tilde{p}_{N}\right\|_{\mathcal{H}_{G_{r}}\left(\mathbb{R}^{n}\right)}\right]$ vanishes. Noting that, in view of point (b) of Proposition 1,

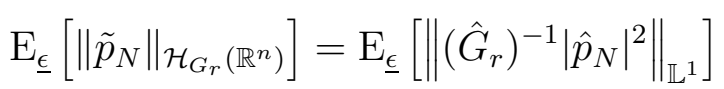

from (44) we conclude that $\mathrm{E}_{\underline{\epsilon}}\left[\left\|\gamma \tilde{p}_{N}\right\|_{\mathcal{H}_{G_{r}}\left(\mathbb{R}^{n}\right)}\right]$ converges to zero. By using point (c) of Proposition 1, this means also that $\mathrm{E}_{\underline{\epsilon}}\left[\left\|\gamma f_{N}-\gamma f\right\|_{\mathcal{H}_{G_{r}}(C)}\right]$ converges to zero. Then the assumptions of Lemma 2 when applied to the sequence $\bar{f}_{N}(\underline{x}, \underline{\epsilon})$ are satisfied and the thesis follows from its use.

Before giving the proof of Theorem 2 we present some technical results that will be useful in handling both the deterministic and the stochastic sampling cases. For the deterministic sampling, we consider multi-indexes $\underline{k}=\left(k_{1}, k_{2}, \ldots, k_{n}\right)$ and the operator $\mathfrak{D}_{K}[F]$ which transforms any Lipschitz, bounded and absolutely integrable function $F:[0,1]^{n} \mapsto[0,1]$ into the function

$$
\mathfrak{D}_{K}[F](\underline{x})=\sum_{k_{1}=0}^{K} \cdots \sum_{k_{n}=0}^{K} F\left(\underline{x}-\frac{\underline{k}}{K}\right) .
$$

Recall that $K$ is defined in Assumption 1 and note that $\mathfrak{D}_{K}\left[G_{r}\right]$ is the denominator of $h_{N}$ when a uniform grid of samples is considered including the origin and with a sampling step $1 /(K-1)$.

Lemma 4. Under Assumption 1 it holds

$$
\lim _{K \rightarrow+\infty} \sup _{[0,1]^{n}}\left\|\left|K^{-n} \mathfrak{D}_{K}\left[G_{r}\right](\underline{x})-\gamma(\underline{x})\right|\right\|_{r-1}=0 .
$$


Proof. To investigate this limit we first analyze

$$
\mathfrak{D}_{K}^{\prime}[G](\underline{x})=\sum_{k_{1}=0}^{\left\lfloor K x_{1}\right\rfloor} \cdots \sum_{k_{n}=0}^{\left\lfloor K x_{n}\right\rfloor} G(\underline{x}-\underline{k} / K)
$$

where $\lfloor\cdot\rfloor$ gives the largest integer not greater than its argument. For a general function $F$ that is Lipschitz, bounded and absolutely integrable, one can show that

$$
\lim _{K \rightarrow \infty} \sup _{[0,1]^{n}}\left|K^{-n} \mathfrak{D}_{K}^{\prime}[F](\underline{x})-\int_{0}^{x_{1}} \cdots \int_{0}^{x_{n}} F\right|=0 .
$$

In fact, note that if $F^{+}=\max \{F, 0\}$ and $F^{-}=\max \{-F, 0\}$, then $\mathfrak{D}_{K}^{\prime}[F]=\mathfrak{D}_{K}^{\prime}\left[F^{+}\right]-$ $\mathfrak{D}_{K}^{\prime}\left[F^{-}\right]$so that we may actually concentrate on non-negative functions $F$. For these functions we indicate with $\chi_{k_{1}, \ldots, k_{n}}$ the indicator function of the $n$-dimensional cell $\left.] k_{1} / K,\left(k_{1}+1\right) / K\right] \times$ $\left.\cdots \times] k_{n} / K,\left(k_{n}+1\right) / K\right]$ and define the function $\hat{F}_{\underline{x}}:\left[0,\left\lceil K x_{1}\right\rceil / K\right] \times \cdots \times\left[0,\left\lceil K x_{n}\right\rceil / K\right] \mapsto$ $\mathbb{R}^{+}$where $\hat{F}_{\underline{x}}(\underline{y})=\sum_{k_{1}=0}^{\left\lfloor K x_{1}\right\rfloor} \cdots \sum_{k_{n}=0}^{\left\lfloor K x_{n}\right\rfloor} F(\underline{x}-\underline{k} / K) \chi_{\left\lfloor K x_{1}\right\rfloor-k_{1}, \ldots,\left\lfloor K x_{n}\right\rfloor-k_{n}}(\underline{y})$ and $\lceil\cdot\rceil$ gives the smallest integer not lower than its argument

With this definition we have

$$
K^{-n} \mathfrak{D}_{K}^{\prime}[F](\underline{x})=\int_{0}^{\left\lceil K x_{1}\right\rceil / K} \cdots \int_{0}^{\left\lceil K x_{n}\right\rceil / K} \hat{F}_{\underline{x}} .
$$

The function $\hat{F}_{\underline{x}}$ can be bounded from above and from below as $\left(F-\frac{L n}{K}\right)^{+} \leq \hat{F}_{\underline{x}} \leq F+\frac{L n}{K}$ where $L$ is the Lipschitz constant such that $\left|F\left(x_{1}^{\prime}, \ldots, x_{n}^{\prime}\right)-F\left(x_{1}^{\prime \prime}, \ldots, x_{n}^{\prime \prime}\right)\right| \leq L \sum_{j=1}^{n}\left|x_{j}^{\prime}-x_{j}^{\prime \prime}\right|$. From this and from (46) we also get

$$
\begin{aligned}
\int_{0}^{\left\lceil K x_{1}\right\rceil / K} \cdots \int_{0}^{\left\lceil K x_{n}\right\rceil / K}\left(F-\frac{n L}{K}\right)^{+} \leq K^{-n} \mathfrak{D}_{K}^{\prime}[F](\underline{x}) & \leq \\
& \leq \int_{0}^{\left\lceil K x_{1}\right\rceil / K} \cdots \int_{0}^{\left\lceil K x_{n}\right\rceil / K} F+\frac{n L}{K} .
\end{aligned}
$$

Since $F$ is bounded, a constant $M>0$ exists such that $0 \leq F \leq M$. Hence, the two integrals in (47) can be bounded to obtain

$$
\begin{aligned}
& \int_{0}^{x_{1}} \ldots \int_{0}^{x_{n}}\left(F-\frac{L n}{K}\right)^{+} \leq K^{-n} \mathfrak{D}_{K}^{\prime}[F](\underline{x}) \leq \\
& \leq M^{n} \prod_{j=1}^{n}\left(\left\lceil K x_{j}\right\rceil / K-x_{j}\right)+\int_{0}^{x_{1}} \ldots \int_{0}^{x_{n}} F+\frac{L n}{K} \leq \\
& \leq\left(\frac{M}{K}\right)^{n}+\int_{0}^{x_{1}} \ldots \int_{0}^{x_{n}} F+\frac{L n}{K} .
\end{aligned}
$$

Since, for $K \rightarrow \infty$, it holds $\left(\frac{M}{K}\right)^{n} \rightarrow 0$, from the Lebesgue Dominated Convergence Theorem it follows that $\int_{0}^{x_{1}} \ldots \int_{0}^{x_{n}}\left(F-\frac{L n}{K}\right)^{+} \rightarrow \int_{0}^{x_{1}} \ldots \int_{0}^{x_{n}} F$ and that, rather obviously, also 
$\int_{0}^{x_{1}} \cdots \int_{0}^{x_{n}} F+\frac{L n}{K} \rightarrow \int_{0}^{x_{1}} \cdots \int_{0}^{x_{n}} F$. From this we know that

$$
\begin{aligned}
\mid K^{-n} \mathfrak{D}_{K}^{\prime}[F](\underline{x})- & \int_{0}^{x_{1}} \ldots \int_{0}^{x_{n}} F \mid \leq \\
& \leq \frac{1}{2}\left[\left(\frac{M}{K}\right)^{n}+\int_{0}^{x_{1}} \ldots \int_{0}^{x_{n}} F+\frac{L n}{K}-\left(F-\frac{L n}{K}\right)^{+}\right] \leq \\
& \leq \frac{1}{2}\left[\left(\frac{M}{K}\right)^{n}+\int_{0}^{1} \ldots \int_{0}^{1} F+\frac{L n}{K}-\left(F-\frac{L n}{K}\right)^{+}\right]
\end{aligned}
$$

where the last inequality exploit the fact that $F+\frac{L n}{K}-\left(F-\frac{L n}{K}\right)^{+} \geq 0$. Yet, this is finally equivalent to (45) as its rhs vanishes independently of $\underline{x}$ when $K \rightarrow \infty$.

Let now $\sigma$ be one of the $2^{n}$ mappings of $\mathbb{R}^{n}$ in itself which do nothing but possibly changing the sign of the axes so that its $j$-th component is either $\sigma_{j}\left(x_{j}\right)=-x_{j}$ or $\sigma_{j}\left(x_{j}\right)=x_{j}$. Let also $\bar{\sigma}$ be a corresponding transformation of $[0,1]^{n}$ into itself whose $j$-th component is such that $\bar{\sigma}_{j}\left(x_{j}\right)=1-x_{j}$ whenever $\sigma_{j}\left(x_{j}\right)=-x_{j}$ and is the identity otherwise. With this we may easily recognize that $\mathfrak{D}_{K}[F](\underline{x})=\sum_{\sigma} \mathfrak{D}_{K}^{\prime}[F \circ \sigma](\bar{\sigma}(\underline{x}))$ so that the $2^{n}$ summands give

$$
\lim _{K \rightarrow \infty} \sup _{[0,1]^{n}}\left|K^{-n} \mathfrak{D}_{K}[F](\underline{x})-\int_{x_{1}-1}^{x_{1}} \ldots \int_{x_{n}-1}^{x_{n}} F\right|=0
$$

Now, we apply the previous result to the derivatives of $M B_{r}$ functions.

It is apparent, from (7) that the first $r-1$ derivatives of $G_{r}$ are Lipschitz, bounded and integrable. As $D^{\alpha} \mathfrak{D}_{K}\left[G_{r}\right]=\mathfrak{D}_{K}\left[D^{\alpha} G_{r}\right]$ we have

$$
\lim _{K \rightarrow \infty} \sup _{\mathbb{R}^{n}}\left|K^{-n} D^{\alpha} \mathfrak{D}_{K}[G](\underline{x})-\int_{x_{1}-1}^{x_{1}} \ldots \int_{x_{n}-1}^{x_{n}} D^{\alpha} G\right|=0 .
$$

The thesis follows from the definition (19) and from the fact that the integral and derivative operators in (49) can be exchanged.

The next two Lemmas provide the necessary tools to analyze uniform convergence issues in the case of stochastic sampling. The first one specialize an elementary analysis result [11].

Lemma 5. Let Assumption 2 hold and let $\phi: \mathbb{R}^{n} \mapsto \mathbb{R}$ be a Lipschitz function. Then, with probability one, it holds

$$
\lim _{N \rightarrow \infty} \sup _{\underline{x} \in C}\left|\frac{1}{N} \sum_{k=1}^{N} \phi\left(\underline{x}-\underline{x}_{k}\right)-\int_{C} \phi(\underline{x}-\underline{\xi}) d \underline{\xi}\right|=0
$$

Lemma 6. Under Assumption 2, for every integer $r \geq 1$, with probability one it holds

$$
\lim _{N \rightarrow \infty}\left\|\frac{1}{N} \sum_{k=1}^{N} G_{r}\left(\underline{x}-\underline{x}_{k}\right)-\gamma(\underline{x})\right\|_{r-1}=0
$$


Proof. For every finite multi-index $\alpha$, such that $|\alpha|_{\infty} \leq r-1, D^{\alpha} G_{r}$ is Lipschitz and also the restriction of $\int_{C} D^{\alpha} G_{r}(\underline{x}-\underline{\xi}) d \underline{\xi}$ to the set $C$ is Lipschitz. Then, Lemma 5 can be applied in order to derive the result.

\section{Proof of Theorem 2.}

Since $f \in \mathcal{H}_{\bar{G}_{r}}(C)$, from (8) we have that $f \in \mathcal{H}_{G_{r}}(C)$, and, from Lemma 3 and proposition $4, \gamma f \in \mathcal{H}_{G_{r}}(C)$.

To shorten notations, we define (both in the deterministic and stochastic sampling cases) $\mathfrak{C}_{N}\left[G_{r}\right]=\sum_{i=1}^{N} G_{r}\left(\underline{x}-\underline{x}_{i}\right)$.

Note that

$$
\begin{aligned}
h_{\rho N} & =\frac{N \bar{R}_{\rho N}}{\mathfrak{C}_{N}\left[G_{r}\right]} \\
\bar{R}_{\rho N}(\underline{x}, \underline{\epsilon}) & =\sum_{k=1}^{N} \bar{b}_{k N}(\epsilon) G_{r}\left(\underline{x}, \underline{x}_{k}\right) \\
\bar{b}_{N}(\epsilon) & =G_{N}^{-1} H_{N}\left[f_{\rho N}\left(\underline{x}_{1}, \underline{\epsilon}\right) \ldots f_{\rho N}\left(\underline{x}_{N}, \underline{\epsilon}\right)\right]
\end{aligned}
$$

where the dependence of the coefficients $\bar{b}_{N}$ on the noise vector $\underline{\epsilon}$ is stressed.

From (50) it follows $\left\|h_{\rho N}-f\right\|_{r-1}=\left\|N \bar{R}_{\rho N} / \mathfrak{C}_{N}\left[G_{r}\right]-f\right\|_{r-1}$ to which we can now add and subtract $N f \gamma / \mathfrak{C}_{N}\left[G_{r}\right]$, apply the triangular inequality and obtain

$$
\mathrm{E}\left[\left\|h_{\rho N}-f\right\|_{r-1}\right] \leq \mathrm{E}\left[\left\|\frac{\bar{R}_{\rho N}-f \gamma}{N^{-1} \mathfrak{C}_{N}\left[G_{r}\right]}\right\|_{r-1}\right]+\mathrm{E}\left[\left\|\frac{f \gamma}{N^{-1} \mathfrak{C}_{N}\left[G_{r}\right]}-f\right\|_{r-1}\right]
$$

To prove that both terms on the right hand side of (53) vanish when $N \rightarrow \infty$ note that, for any two sufficiently smooth functions $\phi_{1}$ and $\phi_{2}$ and any $n$-dimensional multi-index $\alpha \neq(0,0, \ldots, 0),|\alpha|_{\infty} \leq r-1$ we have

$$
D^{\alpha}\left(\phi_{1} \phi_{2}\right)=\sum_{\beta_{1}+\beta_{2}=\alpha} C_{\beta_{1}, \beta_{2}} D^{\beta_{1}} \phi_{1} D^{\beta_{2}} \phi_{2}
$$

where the coefficients $C_{\beta_{1}, \beta_{2}}$ depend only on $\beta_{1}$ and $\beta_{2}$. Setting $\phi_{1}=\bar{R}_{\rho N}-f \gamma$ and $\phi_{2}=$ $N / \mathfrak{C}_{N}\left[G_{r}\right]$, we obtain

$$
\mathrm{E}\left[\left|D^{\alpha}\left(\frac{\bar{R}_{\rho N}-f \gamma}{N^{-1} \mathfrak{C}_{N}\left[G_{r}\right]}\right)\right|\right] \leq \sum_{\beta_{1}+\beta_{2}=\alpha} C_{\beta_{1}, \beta_{2}} \mathrm{E}\left[\left|D^{\beta_{1}}\left(\bar{R}_{\rho N}-f \gamma\right)\right|\right]\left|D^{\beta_{2}}\left(\frac{1}{N^{-1} \mathfrak{C}_{N}\left[G_{r}\right]}\right)\right|
$$

As far as the second term on the right of (53) is concerned, we may set $\phi_{1}=\gamma-N^{-1} \mathfrak{C}_{N}\left[G_{r}\right]$ 
and $\phi_{2}=N f / \mathfrak{C}_{N}\left[G_{r}\right]$ to obtain

$$
\mathrm{E}\left[\left|D^{\alpha}\left(\frac{f \gamma}{N^{-1} \mathfrak{C}_{N}\left[G_{r}\right]}-f\right)\right|\right] \leq \sum_{\beta_{1}+\beta_{2}=\alpha} C_{\beta_{1}, \beta_{2}}\left|D^{\beta_{1}}\left(\gamma-\frac{\mathfrak{C}_{N}\left[G_{r}\right]}{N}\right)\right|\left|D^{\beta_{2}}\left(\frac{f}{N^{-1} \mathfrak{C}_{N}\left[G_{r}\right]}\right)\right|
$$

The last step is to show that the r.h.s. of (54) and (55) asymptotically goes to zero. Hereafter, the proof proceeds in two different ways according to which sampling scheme is considered.

Point a. Under Assumption 1, Corollary 1 can be applied with the choice $f_{N}=f_{\rho N}$. In fact, from (38)-(39) it follows that $\bar{R}_{N}(\underline{x}, \underline{\epsilon})=\bar{R}_{\rho N}(\underline{x}, \underline{\epsilon})$ and that all the assumptions of Corollary 1 are satisfied because $f_{\rho N}(\underline{x}, \underline{\epsilon}) \in \mathcal{H}_{G_{r}}, \forall \underline{\epsilon} \in \mathbb{R}^{N}$ and, from point (a) of Theorem $1, \lim _{N \rightarrow+\infty} \mathrm{E}_{\underline{\epsilon}}\left[\left\|f_{\rho N}(\underline{x}, \underline{\epsilon})-f(\underline{x})\right\|_{G_{r}}^{2}\right]=0$. Then, by using point (a) of Corollary 1, $\mathrm{E}_{\underline{\epsilon}}\left[\left\|\bar{R}_{\rho N}-f \gamma\right\|_{G_{r}}^{2}\right]$ asymptotically goes to zero. Moreover, from (10), also $\mathrm{E}\left[\left\|\bar{R}_{\rho N}-f \gamma\right\|_{r-1}\right]$ vanishes. It follows that the r.h.s. of (54) asymptotically vanishes as the first $r-1$ derivatives of $N / \mathfrak{C}_{N}\left[G_{r}\right]$ is bounded (and the bound does not depend on $N$ ). Finally, the r.h.s. of (55) converges to zero by virtue of Lemma 4.

Point $b$. As for point (a), we can apply Corollary 1 with the choice $f_{N}=f_{\rho N}$. Since Assumption 2 holds, from point (b) of Corollary 1, we have that $\mathrm{E}_{\underline{\epsilon}}\left[\left\|\bar{R}_{\rho N}-f \gamma\right\|_{G_{r}}^{2}\right]$ asymptotically goes to zero with probability one. Then, from (10), also $\mathrm{E}\left[\left\|\bar{R}_{\rho N}-f \gamma\right\|_{r-1}\right]$ vanishes. It follows that the r.h.s. of (54) asymptotically vanishes with probability one as the first $r-1$ derivatives of $N / \mathfrak{C}_{N}\left[G_{r}\right]$ is bounded (and the bound does not depend on $N$ ). To what concerns the r.h.s. of (55), convergence to zero with probability one is guaranteed by Lemma 6.

\section{References}

[1] R. A. Adams. Sobolev Spaces. Academic Press, New York, 1975.

[2] N. Aronszajn. Theory of reproducing kernels. Trans. Amer. Math. Soc., 68:337-404, 1950.

[3] M. Bertero. Linear inverse problems and ill-posed problems. Advances in Electronics and Electron Phys., 75:1-120, 1989.

[4] G.D. Birkhov. Proof of the ergodic theorem. Proc. of the National Academy of Science, USA, 17:656-660, 1931.

[5] J.J. Buckley. Universal fuzzy controllers. Automatica, 28:1245-1248, 1992.

[6] V. Corradi and H. White. Regularized neural networks: some convergence rate results. Neural Computation, 7:1225-1244, 1995.

[7] P. Craven and G. Wahba. Smoothing noisy data with spline functions: estimating the correct degree of smoothing by the method of generalized cross-validation. Numer. Math., 31:377-403, 1979.

[8] G. De Nicolao and G. Ferrari-Trecate. Consistent identification of NARX models via regularization networks. IEEE Trans. Autom. Contr., 44(11):2045-2049, 1999. 
[9] C. Fantuzzi and R. Rovatti. On the approximation capabilities of the homogeneous takagi-sugeno model. In Fifth IEEE International Conference on Fuzzy Systems, pages 1067-1073, New Orleans, USA, 1996.

[10] G. Ferrari-Trecate and R. Rovatti. Sobolev approximation by means of fuzzy systems with overlapping gaussian concepts. In Eighth IEEE International Conference on Fuzzy Systems, Seoul, Korea, 1999.

[11] G. Ferrari-Trecate and R. Rovatti. Sobolev approximation by means of fuzzy systems with overlapping gaussian concepts. Fuzzy sets and systems, 2001. To appear.

[12] F. Girosi. An equivalence between sparse approximation and support vector machines. Neural Computation, 10:1455-1480, 1998.

[13] F. Girosi, M. Jones, and T. Poggio. Regularization theory and neural networks architectures. Neural Computation, 7:219-269, 1995.

[14] E.S. Gradshteyn and I.M. Ryzhik. Table of Integrals, Series and Products (fifth ed.). Academic Press, New York, 1993.

[15] S. Haykin. Neural networks - a comprehensive foundation. Macmillan, Englewood Cliffs, 1994.

[16] T.A. Johansen. Robust identification of takagi-sugeno-kang fuzzy models using regularization. Fifth IEEE International Conference on Fuzzy Systems, pages 180-186, 1996.

[17] B. Kosko. Fuzzy systems as universal approximators. IEEE Transactions on Computers, 43:1329-1330, 1994.

[18] Yi Lin. Tensor product space ANOVA models. The Annals of Statistics, 28(3):734-755, 2000.

[19] D.J.C. MacKay. Bayesian interpolation. Neural Computation, 4:415-447, 1992.

[20] B. Matérn. Spatial Variation (second ed.). Springer-Verlag. Lecture Notes in Statistics, 36, 1986.

[21] R.M. Neal. Bayesian Learning for Neural Networks, volume 118 of Lecture Notes in Statistics. Springer-Verlag, New York, 1996.

[22] J. Park and I. W. Sandberg. Universal approximation using radial-basis-function networks. Neural Computation, 3:246-257, 1991.

[23] T. Poggio and F. Girosi. Networks for approximation and learning. IEEE Proc., 78:14811497, 1990.

[24] F. Riesz and B. Sz.-Nagy. Functional Analysis. Frederick Ungar, New York, 1955.

[25] R. Rovatti. Takagi-sugeno models as approximators in Sobolev norms: the SISO case. In Fifth IEEE International Conference on Fuzzy Systems, pages 1060-1066, New Orleans, USA, 1996.

[26] R. Rovatti. Fuzzy piecewise-multilinear and piecewise-linear systems as universal approximators in Sobolev spaces. IEEE Transactions on Fuzzy Systems, 3:235-249, 1998.

[27] R. Rovatti, C. Fantuzzi, S. Simani, and S. Beghelli. Parameters identification for piecewise linear models with weakly varying noise. In IEEE International Conference on Decision and Control (CDC'98), 1998.

[28] S. Simani, C. Fantuzzi, R. Rovatti, and S. Beghelli. Noise rejection in parameters identification for piecewise linear fuzzy models. In Seventh IEEE International Conference on Fuzzy Systems, Anchorage, USA, 1998.

[29] S. Simani, C. Fantuzzi, R. Rovatti, and S. Beghelli. Parameter identification for 
piecewise-affine fuzzy models in noisy environment. International Journal of Approximate Reasoning, 22, 1999.

[30] A.N. Tikhonov and V.Y. Arsenin. Solutions of ill-posed problems. Winston/Wiley, Washington, 1977.

[31] F. Vivarelli. Studies on generalization in Gaussian processes and Bayesian Neural Networks. PhD thesis, Neural Computing Research Group, Aston University, Birmingham, United Kingdom, 1998.

[32] G. Wahba. Practical approximate solutions to linear operator equations when the data are noisy. SIAM J. Numer. Anal., 14:651-667, 1977.

[33] G. Wahba. Spline Models for Observational Data. CBMS-NSF Regional Conference Series in Applied Mathematics. Vol. 59. SIAM, Philadelphia, 1990.

[34] C.K.I. Williams and F. Vivarelli. Upper and lower bounds on the learning curve for gaussian processes. Technical Report NCRG/98/015, Aston University, Birmingham, UK, 1998.

[35] C.K.J. Williams and C.E. Rasmussen. Gaussian processes for regression. In D.S. Touretzky, M.C. Mozer, and M.E. Hasselmo, editors, Advances in Neural Information Processing Systems, 8. MIT Press, 1996.

[36] X.-J. Zeng and M.G. Singh. Approximation theory of fuzzy systems - SISO case. IEEE Transactions on Fuzzy Systems, 2:162-176, 1994.

[37] X.-J. Zeng and M.G. Singh. Approximation theory of fuzzy systems - MISO case. IEEE Transactions on Fuzzy Systems, 3:219-235, 1995. 